\title{
Open Otto Chip as an SPR Pressure Transducer
}

\author{
J. O. Maciel Neto, ${ }^{1}$ Gustavo Oliveira Cavalcanti, ${ }^{2}$ Ignacio Llamas-Garro, ${ }^{3}$ Jung-Mu Kim ${ }^{4}$ and Eduardo Fontana ${ }^{5}$ \\ ${ }^{1}$ Instituto Federal de Pernambuco, Recife, Brazil \\ ${ }^{2}$ Escola Politécnica, Universidade de Pernambuco, Recife, Brazil \\ ${ }^{3}$ Centre Tecnològic de Telecomunicacions de Catalunya, Castelldefels, Spain \\ ${ }^{4}$ Division of Electronic Engineering, Chonbuk National University, Jeonju 561-756, Republic of Korea \\ ${ }^{5}$ Departamento de Eletrônica e Sistemas, Universidade Federal de Pernambuco, Recife, Brazil
}

\begin{abstract}
A sealed Otto chip device has recently been demonstrated as a potential alternative for the traditional Kretschmann based configuration for the development of surface plasmon resonance - SPR - sensors. We have fabricated an open Otto chip structure on silicon and have characterized the SPR effect at a wavelength of $975.1 \mathrm{~nm}$. In this paper we report on the potential of the structure to act as a pressure transducer.
\end{abstract}

Keywords-Otto chip; pressure; silicon; gold; SPR

\section{INTRODUCTION}

We have recently reported on the fabrication of an Otto chip device [1], capable of exhibiting the surface plasmon resonance SPR - effect [2]-[3]. The device comprises a closed structure having a well-defined gap distance between the inner surface of a glass window and the metal surface. By use of the traditional prism coupling configuration [2]-[3], an input light beam can be used to excite a surface plasmon on the metal surface. The critical step in fabricating such structure is the sealing without offsetting the gap distance, which is a critical parameter to define the degree of coupling between the incoming beam and the surface plasmon on the metal [1].

To further investigate the properties of these Otto chip structures, we have also fabricated open devices, i.e., devices without the glass window, to explore potential applications of the SPR effect in an open configuration. One of these studies comprises evaluating the possibility of using the structure as a pressure transducer, as the gap distance affects the degree of coupling of the input beam with the surface plasmon on the metal surface. In this paper we report on preliminary studies directed to this application.

\section{OpEN OTTO ChIP FABRICATION}

A silicon wafer was used as substrate to fabricate several devices. First, an initial cavity with a depth of $2.5 \mu \mathrm{m}$ was formed on the silicon substrate using a deep reactive ion etching (DRIE) process [1], with a thin positive photoresist (PR) used as an etch mask, as illustrated in Fig.1. Then, the PR was removed using an oxygen plasma etcher. A $\mathrm{Cr}$ film of $10 \mathrm{~nm}$ was sputtered on the silicon surface, followed by 300 $\mathrm{nm}$ of Au. After metal deposition, the metal coating was patterned inside the silicon cavity using the lift-off process, by use of AZ5214 as a PR mold [4]. The backside of the

This work was sponsored by the following agencies: Conselho Nacional de Desenvolvimento Científico e Tecnológico (CNPq - Brazil) (56066520105 and 45983620145); NRF-Korea (NRF-2013K2A1A2049144), MINECO-Spain (PIB2010BZ-00585); GENCAT-Spain (2014 SGR 1551). (a)

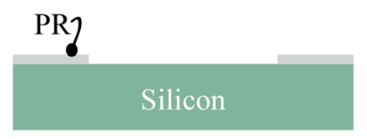

(b)

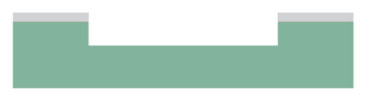

(f)

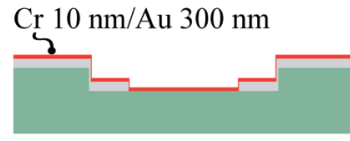

(c)

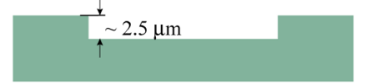

(g)

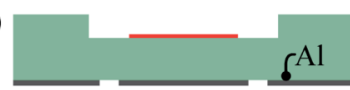

(d)

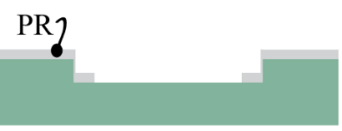

(h)

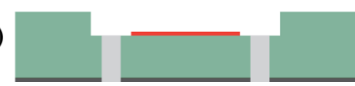

Fig. 1. (a) PR is patterned on $\mathrm{Si}$ substrate. (b) A $2.5 \mu \mathrm{m}$ cavity is produced by DRIE and (c) PR is removed. (d) Positive PR added. (e) Metallization and (f) PR removal. (g) Al mask is produced on the back and (h) channels are etched through Al mask.

silicon wafer was etched by use of a Bosch DRIE process [5], to yield the inlet and outlet of the channel of the chip, with aluminum used as the etch mask. The devices thus fabricated were diced into $30 \mathrm{~mm}$ x $30 \mathrm{~mm}$ open chips.

\section{EXPERIMENTAL SET-UP}

The Otto chip response was measured by use of an automated reflectometer operating at a wavelength of 975.1 $\mathrm{nm}$ [6], as illustrated in Fig.2. A right angle BK7 prism is used to allow coupling the plane-polarized laser beam to a surface plasmon on the inner metal surface of the chip. The SPR effect is observed in the angular distribution of the reflected

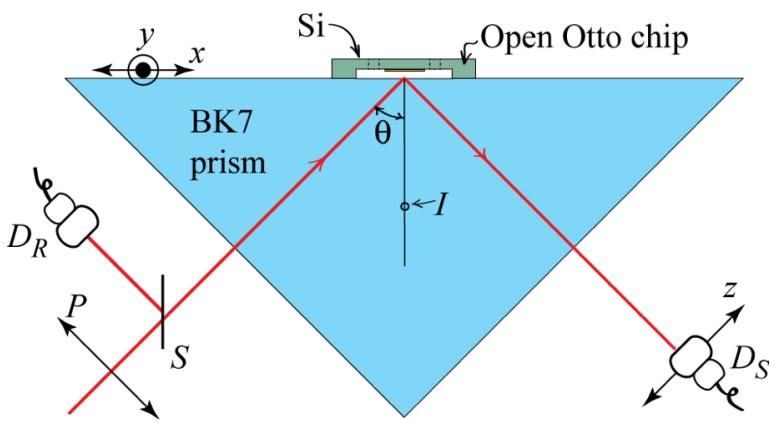

Fig. 2. Experimental set-up for measuring the open Otto chip response. In the figure: $D_{R}=$ reference photodetector, $D_{S}=$ signal photodetector, $S=$ splitter, $P=$ polarizer, $I=$ right angle prism's incenter. 
intensity, recorded by photodetector $D_{S}$. Fluctuations in the intensity of the input beam are eliminated by simultaneous measurement of a sample of the input beam, by a reference photodetector $D_{R}$, illustrated in the figure. The prism angle and horizontal position along two orthogonal directions in the $x y$ plane shown in the figure are computer controlled. The system has a built-in control so as to maintain the laser footprint stationary, independently or translation or rotational movementes [7].

In order to keep the Otto chip attached to the prism surface, a rubber buffer was used, as illustrated in Fig.3a. A gas cell, shown in Fig. $3 \mathrm{~b}$ was used to both press the buffer against the Otto chip and to seal the Otto chip environment, upon which a small vacuum or small pressure could be applied. By careful adjustment of the cell's screws shown in Fig. $3 b$, a given set point of the gap between the gold surface of the Otto chip and the prism surface could be achieved. From this point, sub micrometric changes of the gap distance were produced by changing the pressure level within the cell by inserting/removing $N_{2}$ gas from a gas manifold (not shown in the figures).

\section{RESUlTS}

Different pressure levels were applied within the gas cell shown in Fig.3. Figure 4 shows resonance curves obtained at three pressure levels within the cell. It is important to notice that because the gap thickness is approximately $2.2 \mu \mathrm{m}$, even small pressure differences within the cell may produce

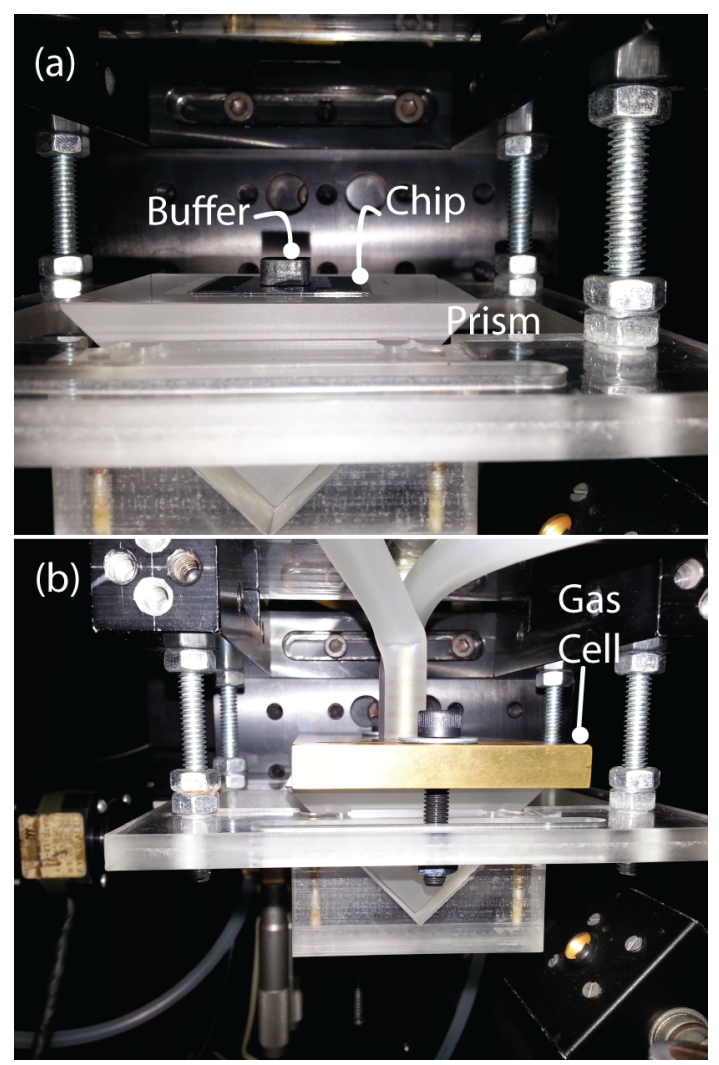

Fig. 3. Photographs of the prism/chip/cell configuration. (a) Details of the buffer/chip positioning on the prism. (b) Details of the gass cell on the prism.

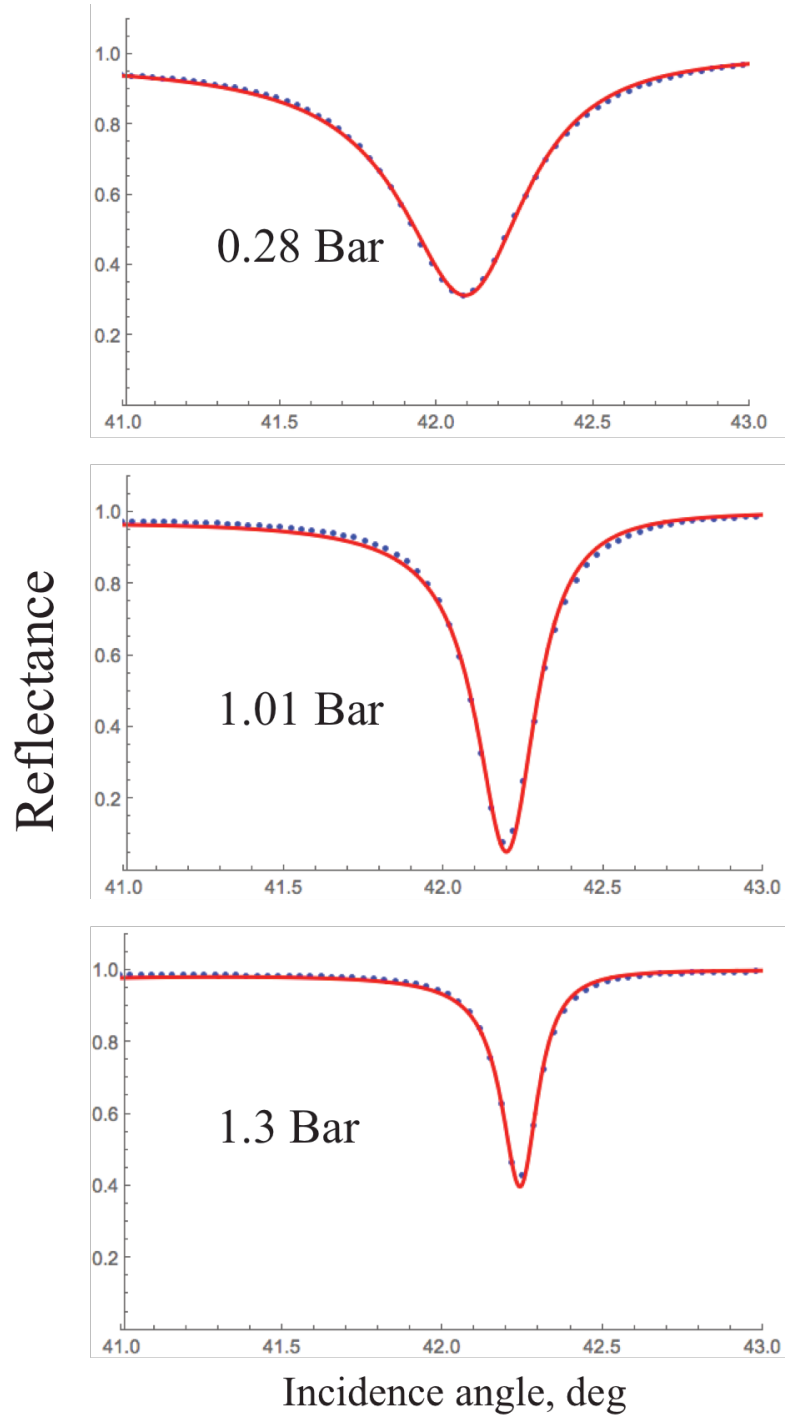

Fig. 4. SPR curves for 3 distinct pressure levels within the Otto chip.

substantial changes in the gap distance between the metal surface and the prism surface in the configuration shown in Fig.2. In the plots of Fig.4, the dots are the measured values and the solid lines are the theoretical curves obtained by nonlinear regression analysis of the data [8]. For the analysis the small change in refractive index of the cell due to the distinct gas pressures was neglected. From this analysis, the complex refractive index of the metal and the gap thickness were obtained. The complex refractive index was $\bar{n} \approx 0.2-$ $j 6$, in agreement with the value obtained previously and with the tabulated value for gold at $975.1 \mathrm{~nm}$ [9]-[10]

Figure 5 is a plot of the gap thickness as a function of the cell pressure, showing an approximate linear relationship in the range 0.3 to 1.3 Bar. In this range, the gap thickness changed from 1.3 to $2.5 \mu \mathrm{m}$ approximately.

\section{CONCLUSIONS}

In this paper we have investigated the sensitivity of the Otto chip to pressure changes within the gap of the device. 
The results show that in the open configuration, the gap distance is a very sensitive function of the pressure and this may allow using this technique to develop optical pressure transducers. The working pressure range of the transducer can be tailored by using different buffer materials.

In many situations, it is important to carry out remote measurements of environmental pressure, e.g., in process control, with a passive sensor head, without use of electronic transmission of the signal. In these cases, an all optical solution may be employed with conjunction with optical fibers. In these situations, an optical transducer of the type proposed in this paper may be employed.

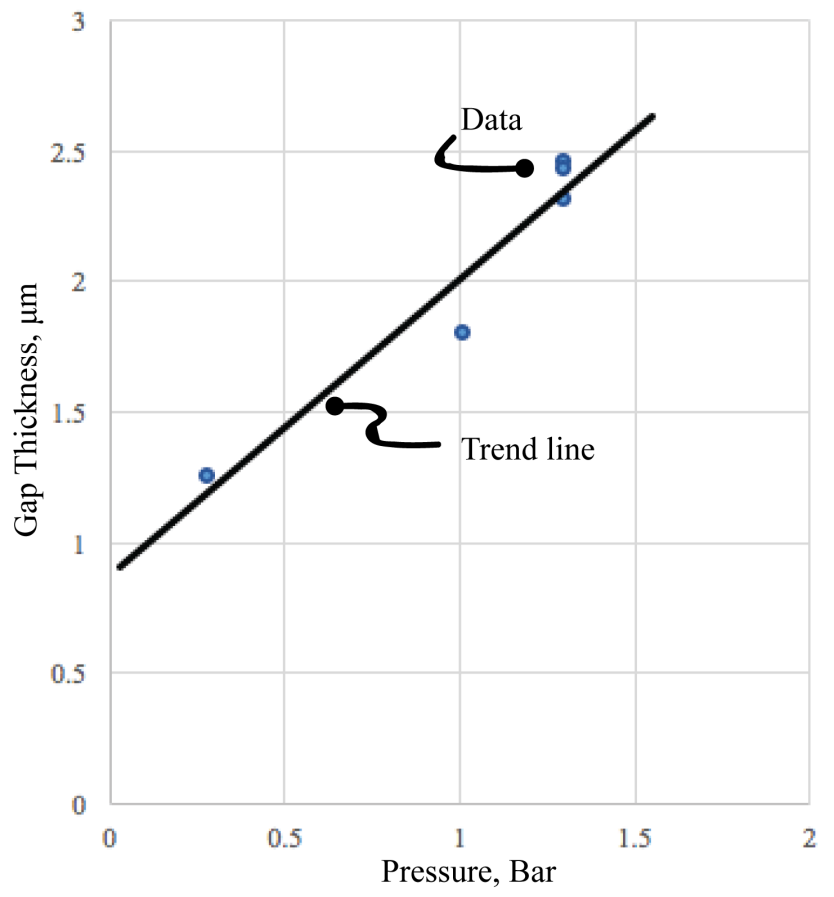

Fig. 5. Measured gap thickness as a function of the gas pressure.

\section{REFERENCES}

[1] Eduardo Fontana, Jung-Mu Kim, Ignacio Llamas-Garro and Gustavo Oliveira Cavalcanti, "Microfabricated Otto chip device for surface plasmon resonance based optical sensing," Applied Optics, vol. 54, pp.9200-9204, November 2015.

[2] A. Otto," Excitation of Nonradiative Surface Plasma Waves in Silver by the Method of Frustrated Total Reflection," Z. Physik 216, 398-410 (1968).

[3] E. Kretschmann, "Determination of optical constants of metals through the stimulation for surface plasma oscillations," (in German), Z. Physik 241, 313-324 (1971).

[4] H. W. Park, Y. K. Kim, H. G. Jeong, J. W. Song, and J. M. Kim, "Feedthrough capacitance reduction for a micro-resonator with push-pull configuration based on electrical characteristic analysis of resonator with direct drive," Sensors and Actuators A, Vol. 170, No. 1, pp. 131138 (2011).

[5] Y. S. Lee, Y. H. Jang, Y. K. Kim, and J. M. Kim, "Thermal de-isolation of silicon microstructures in a plasma etching environment," Journal of Micromechanics and Microengineering, Vol.23, No. 2, 025026 (2013).

[6] G. O. Cavalcanti, M. A. Luna and E. Fontana, "Automated reflectometer for surface plasmon resonance studies in the infrared and its application for the characterization of Pd films," Proceedings of the SBMO/IEEE MTT-S International Microwave and Optoelectronics Conference, pp. 688-701 (2007).

[7] E. Fontana and G. O. Cavalcanti, "Maintaining a stationary laser footprint during angular scan in internal reflection experiments," Applied Optics, Vol. 52, No. 32, pp. 7669-7674 (2013).

[8] E. Fontana, R. H. Pantell and M. Moslehi, "Characterization of dielectric-coated, metal mirrors using surface plasmon spectroscopy," Applied Optics 27, 3334-3340 (1988).

[9] Glenn D. Boreman, Timothy Johnson, Andrew C. Jones, Sang-Hyun Oh, Robert L. Olmon, Markus B. Raschke, David Shelton, and Brian Slovick, "Broadband Electrical Permittivity of Gold for Plasmonics and Nano-Optics Applications," 2011 Conference on Lasers and ElectroOptics (CLEO 2011), pp.1-2.

[10] P. B. Johnson and R. W. Christy, "Optical constants of the noble metals," Physical Review B, Vol. 6, No. 12, pp. 4370-4379 (1972) 